\title{
WEBOMETRIA E OS PERIÓDICOS CIENTÍFICOS ELETRÔNICOS
}

Milton Shintaku*

RESUMO As métricas para avaliar periódicos científicos têm sido utilizadas por várias disciplinas, não somente na biblioteconomia ou ciência da informação, visto que os periódicos são canal de comunicação utilizado por todas as disciplinas para disseminar os resultados de pesquisa. Entretanto, não há um consenso de qual a melhor métrica para sua avaliação, visto as diferenças disciplinares, evolução tecnológica e mudanças nos processos de publicação. Assim, o presente estudo tem por objetivo revisar os conceitos e aplicações da webometria, no âmbito dos periódicos científicos. Com isso, contribuir com a discussão atual sobre indicadores. $O$ estudo revela que os inlinks de uma revista eletrônica online são citações em sua grande parte, alinhando a bibliometria, mas podem também ser indicação de artigos em redes sociais, aproximando-se da altimetria. Entretanto, estudos baseados somente nos links não fornecem a acurácia necessária às métricas, de forma que estudos mais profundos, de caráter qualitativo, são requeridos para o desenvolvimento de melhores indicadores, visto os problemas envolvendo os links.

Palavras-chave: Bibliometria. Revista eletrônica. Estudos métricos. Revistas científicas. Indicadores webométricos.

* Doutor em Ciência da Informação pela Universidade de Brasília, Brasil. Tecnólogo do Instituto Brasileiro de Informação em Ciências e Tecnologia, Brasil. Professor da Secretaria de Educação do Distrito Federal, Brasil.

E-mail: milton.shintaku@gmail.com.

\section{INTRODUÇÃO}

P ode se afirmar que o surgimento dos periódicos científicos eletrônicos on-line impactou profundamente os processos envolvidos na publicação de resultados de pesquisa. Meadows (2001), refletindo sobre a transição do impresso ao digital, advoga que o processo de publicação online se torna mais diversificada e mais globalizada, tornando mais fácil o acesso, mudando o papel de vários atores nesse processo, na medida em que surgem novas atividades.

Não apenas o processo de publicação foi alterado pelo periódico eletrônico on-line, mas também outros aspectos envolvidos com a comunicação científica são afetados, tanto que Cruz et al (2003) descrevem os impactos dessa modalidade de publicação nas bibliotecas universitárias, revelando a mudança de posicionamento da detenção da propriedade para a oferta do acesso dos conteúdos. Com a mudança do físico para o digital com acesso online outras questões também se evidenciam, por exemplo a preservação do artigo em si ou do seu acesso, a colaboração pós publicação e outros (STUMPF, 1996).

Nesse contexto, ainda existem algumas questões relevantes que são discutidas em relação ao ambiente virtual e os periódicos científicos como as métricas, na medida em que indicadores tradicionais bibliométricos podem não ser mais tão representativos nesse novo cenário. Mesmo assim, Lopes et al (2012) advoga que novos indicadores bibliométricos, assim como ferramentas para extraí-los, são indispensáveis para medir a qualidade e impacto das publicações e seus autores, mas que requerem cuidado e conhecimento para sua utilização.

Por exemplo, Silva e Bianchi (2001) defendem o uso de indicadores bibliométricos para visão da ciência, da produção científica, mas relatam as suas limitações, destacando que para avaliação pontual, como produção de um 
pesquisador, torna-se menos fidedigna, pois possuem poucos dados para análise. Assim, Nascimento e Oddone (2015) advogam pelo uso da altimetria como opção de métrica para avaliação de citação, na medida em que incorpora fontes não formais e sua relação com as mídias e redes sociais.

Como os periódicos científicos online estão disponíveis na Web, outra opção de métrica é a Webometria, termo que foi cunhado por Almind e Ingwersen (1997), de forma a analisar as relações entre as páginas web, com uso de indicadores quantitativos infométricos. De forma a complementar estes indicadores, Björneborne Ingwersen (2004) utilizaram as abordagens bibliométricas para verificar a construção e uso de recursos, estrutura e tecnologias Web, no âmbito da webometria. Assim, tem-se uma opção diferente para a análise dos periódicos científicos na web, de forma mais abrangente, que envolve Informetria e bibliometria.

Ninkov e Vaughan (2017) verificaram que técnicas webometricas são úteis na análise de informação em variadas fontes, mesmo que tenham se restringido ao tópico de saúde pública. Já Stuart, Stuart e Thewall (2017) verificaram o uso dessas mesmas técnicas para verificação de informação sobre universidades no Instagram, focado em imagens. Por sua vez, Kousha e Thewall (2017a) estudaram o uso da popularização da ciência, utilizando técnicas webometricas em reportagens da British Broadcast Company - BBC.

Ingwersen (2017) afirma que estudos infométricos e webométricos não podem ser desprezados, na medida em que a recuperação da informação na web, atualmente, é dependente de motores de busca. Da mesma forma as ferramentas webométricas podem ser utilizadas para verificar a visibilidade de artigos e livros na disseminação, divulgação e ensino, tanto que Kousha e Thewall $(2017 b, 2017 \mathrm{c})$ estudaram as citações no Wikipedia e Researchgate, verificando certo alinhamento destas com as citações apresentadas no Scopus.

Desta forma, revela-se então a pertinência dos estudos webométricos em sistemas web, apropriado às pesquisas mais amplas dos periódicos científicos on-line disponíveis na web. A partir disso, busca-se estudar as relações dos periódicos e seus artigos com todo o ambiente web, por meio dos seus links. Entende- se que a webometria tem limitações, como apresentado por Vanti (2002), no qual os estudos webométricos estão contidos na informetria e compartilham conhecimentos com a bibliometria e cientometria.

Entretanto, devido aos periódicos científicos estarem acessíveis na web, suas relações webométricas se tornam relevantes para entender certos fenômenos, na medida em que estudos de webometria em periódicos científicos ainda se apresentam tímidos, muito provavelmente por questões voltados ao fator de impacto dominante nas avaliações. Assim, o presente trabalho aplica os conceitos e indicadores webométricos aos periódicos científicos, de forma a verificar a sua aplicação para avaliação.

\section{METODOLOGIA}

O presente estudo possui características teóricas, na medida em que atualiza os conceitos e aplicações da webometria para os periódicos científicos. Assim, a webometria é utilizada como método, amparado pela base conceitual composto pelas relações de links proposta por Thewall (2008) e Björnborn e Ingwersen (2004), além dos estudos webométricos de Aguillo et al (2008, 2010 e 2011).

Lorentzen (2014), comparando o método webométrico e o webmining, destaca que a webometria possui maior foco na estrutura e conteúdo de sites web, sendo apropriado aos estudos das ciências sociais, como nos estudos exploratórios, enquanto o webmining se aproxima de mineração de dados na web. Zahran et al (2014) relata que métodos webométricos, principalmente o Fator de Impacto Web, podem ser utilizados para gerar indicadores de visibilidade de um site, mesmo que não possibilite gerar conclusões gerais.

A webometria, como área de estudo e método, é alinhada aos preceitos da Ciência da Informação (CI), na medida em que a Web se torna uma fonte de informação importante, como as análises de citações de web, a classificação e medição de pesquisas científicas e produtividade de patentes (IBRAHIM, 2013). Para tanto, os estudos webométricos fazem uso de links entre sites, classificando-os como apresentado por Thewall, Vaughan e Björnborn (2005), sendo de forma sumarizada conforme a quadro 1. 
Quadro 1 - Relacionamento entre sites por meio de links

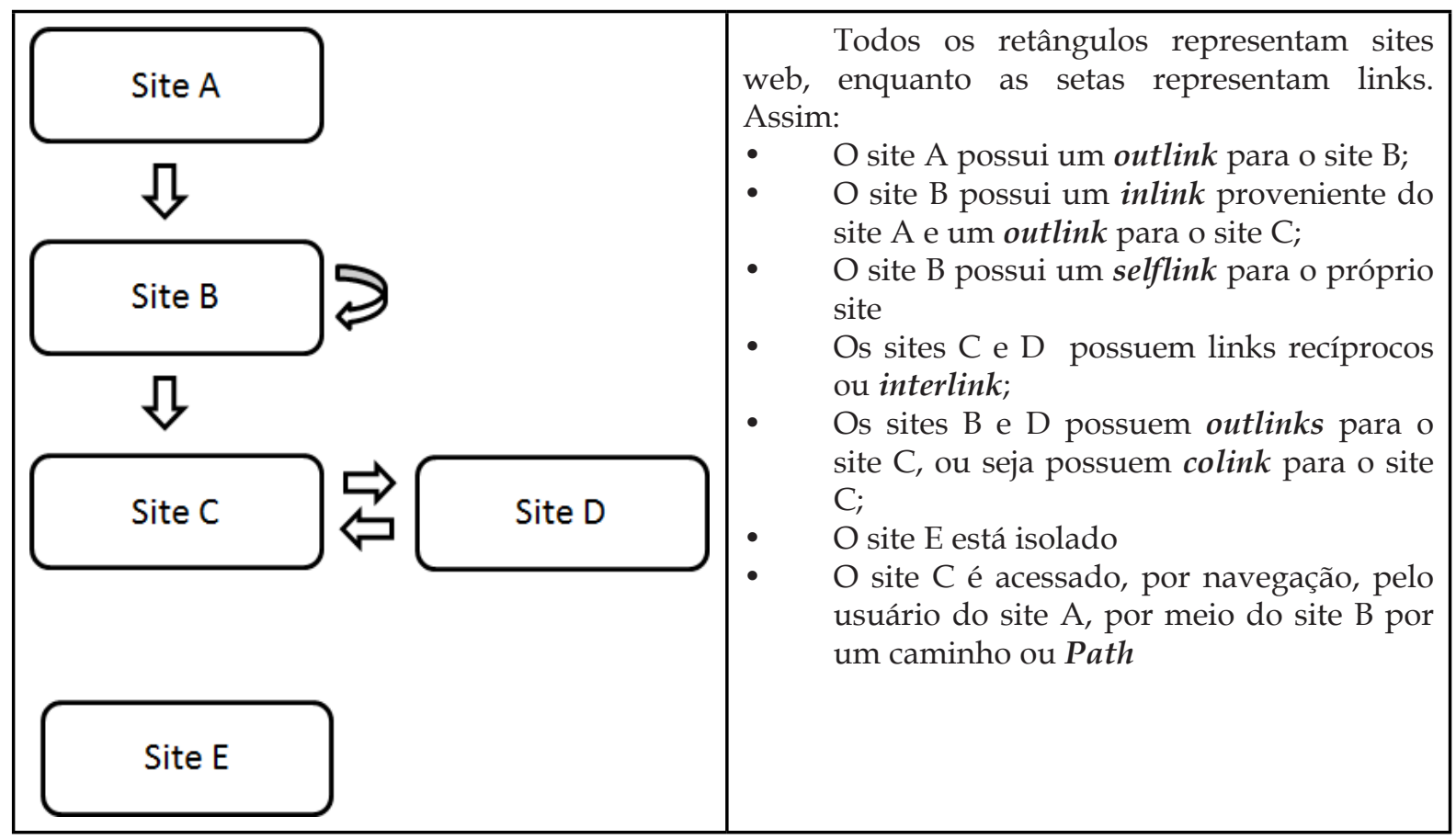

Fonte: Adaptado de Thelwall, Vaughan e Björnborn (2005).

A análise da presença desses tipos de links possibilitam a verificação das estruturas internas e externas de um site, explorando suas relações, de forma a descobrir material útil para fins científicos (BJÖRNBORN; INGWERSEN, 2004). Compreender o comportamento dos sites e seus links pode também apoiar estudos de navegação, para recuperação da informação em diferentes contextos.

$\mathrm{O}$ estudo avalia o uso de conceitos e indicadores de revistas implementados com o OJS, visto que, segundo Shintaku, Brito e Carvalho Neto (2014) há mais de 1600 revistas utilizando essa ferramenta no Brasil, sendo o software livre mais utilizado para criação de periódicos científicos no país.

Cabe notar que no Open Journal System, todos os recursos possuem URL própria. Entretanto, pode-se restringir na seguinte estrutura: URL da revista, marcando pela página inicial; URL da edição, marcado pela página de sumário e URL do artigo, marcado pela página de identificação do artigo.

Para a coleta de dados utilizou-se o Google e Google Acadêmico, pois como afirma Aguillo (2011), essas ferramentas se apresentam apropriadas a estudos webométricos, mesmo com certas restrições técnicas. Os endereços WEB (URL) das revistas são colocados no google para verificar quais sites os referenciam, de forma a verificar o comportamento. Assim, para cada indicador webométrico tem-se uma coleta e análise.

\section{ANÁLISE}

O estudo levanta indicadores webométricos apresentados pela literatura de forma a verificar as ocorrências possíveis. Com isso, estabelece e discute as possibilidades de relações webométricas, na medida em que o cenário se apresenta amplo, com uma grande quantidade de revistas. Apresenta-se um modelo que pode ser verificado pelas revistas, levantando então a frequência para cada caso.

Ao analisar o comportamento dos links em relação a revistas OJS referentes aos dois principais indicadores webométricos (inlinks e outlinks) surge a questão de visibilidade e relacionamentos. Se inicialmente os links entre sites foram considerados comunicações informais, mais atualmente estes podem ser considerados como indicadores importantes de avaliação (THELWALL; VAUGHAN; BJÖRNBORN, 2005; AGUILLO et al, 2010). Assim, os resultados de tipos de links encontrados pelo estudo em revistas foram sintetizados no quadro 2. 
Quadro 2 - Quadro resultado das buscas por URL das revistas

\begin{tabular}{|l|ll|}
\hline \multicolumn{1}{|c|}{ Indicadores } & \multicolumn{2}{c|}{ Ocorrências } \\
\hline Inlinks & $\begin{array}{l}\text { 1. } \\
\text { a. }\end{array}$ & Para a Revista \\
de pós-graduação e outros; \\
b. & Página de redes sociais da revista (twiter, facebook, etc). \\
& C. & Posts em redes sociais convidando a conhecer a revista \\
& 2. & Para a Edição \\
& a. & Post nas redes sociais sobre o lançamento de um novo número da revista \\
& 3. & Para o Artigo \\
& a. & Referência de outros artigos para um determinado artigo \\
& b. & Post em redes sociais sobre um determinado artigo da revista \\
& C. & Repositórios institucionais indicando a origem do artigo \\
& d. & Páginas indicando o artigo como referência \\
\hline Outlink & 1. & Links para órgãos de fomento \\
& 2. & Links para bases indexadoras \\
& 3. & Links para bases de dados \\
& 4. & Links para universidades, associações e outros mantenedores da revista \\
& 5. & Links de artigos referenciando outros documentos \\
\hline
\end{tabular}

Fonte: os autores (2017)

Cabe esclarecer que nesse cenário, o Digital Object Identifier (DOI) se apresenta como um selflink, pois é um outlink que envia para a base de dados DOI, retornando como link ao artigo, revista ou fascículo, por isso, não relevante ao estudo. Verificou-se, também, que as mídias sociais se tornaram importante canal de divulgação, em que muitas revistas criam sua página no Facebook para apoiar a divulgação, encontrando alinhamento com tendências atuais como relatado por Manca e Ranieri (2017) e os achados de NG (2017). Menos comum, mas começando a ter espaço nas revistas está o Youtube, como forma de disponibilizar vídeos de apresentação do artigo. Além disso, existem os casos de blogs acadêmicos (SHEMA; BAR-ILAN; THELWALL, 2014a; SHEMA; BAR-ILAN; THELWALL, 2014b) e de portais de notícias ou divulgação científica (LOOSE; LIMA, 2013; PORTO, 2013;) que referenciam as revistas. Assim, links da revista nessas mídias sociais estão presentes e podem representar visibilidade ou referências.

Em um contexto mais genérico, as revistas online são basicamentes sites web, com várias páginas, em um domínio próprio. Assim, pode-se verificar o fator de impacto web (WIF), do original inglês Web Impact Factor, um indicador derivado dos conceitos bibliométricos de citação denominado fator de impacto, como relata Ingwersen (1998). Assim, inicialmente o WIF era calculado pelo número de inlinks mais os selflinks, dividido pelo número de páginas, sendo posteriormente revisto por Noruzi (2006) como o número de inlinks pelo número de páginas.

Assim, pode-se utilizar esse índice para verificar o impacto web da revista, de forma geral ou particularizada, de forma a analisar o impacto da revista como um todo, de um determinado volume ou de um determinado artigo, pois todo recurso possui um link distinto, mas todos sob um mesmo domínio. Da mesma forma pode-se verificar se os inlinks são provenientes de outras revistas (citações) ou de redes e mídias sociais (altimetria).

Aguillo, Ortega e Fernandes (2008) adaptaram indicadores webométricos, com pesos, para ranquear universidades, no qual pode-se ajustar para avaliar os periódicos científicos da seguinte forma:

a) Tamanho (size): número de páginas indexadas por motores de busca, de forma a ser recuperável, indicando a quantidade de informação publicada, podendo possuir os seguintes subindicadores:

- Número de páginas indexadas: no caso das revistas tem relação com a produtividade, ou seja, a quantidade de artigos publicados;

- Número de arquivos em vários formatos e em inglês: revistas que publicam seus arti- 
gos em mais que um formato (HTML, XML, PDF, E-Pub e outros) aumentam a quantidade de links da revista, com a maior possibilidade de achar o artigo, da mesma forma que as revistas que publicam artigos em inglês.

b) Visibilidade: número de inlinks a partir de locais externos. Em revistas estão mais ligados aos inlinks relacionados às citações dos artigos publicados, com isso aproxima-se da bibliometria. Nos casos dos inlinks provenientes das redes sociais, estes têm aspecto mais associados à altimetria. Um ponto contra a questão da visibilidade por meio da contagem dos inlinks está na citação sem a informação do link, ou seja, leem o artigo digital, mas citam-no como se fosse impresso.

c) Luminosidade: número de outlinks para sites externos. No caso das revistas ainda não é comum ter muitos links, sendo colocado mais nos rodapés, para indexadores, órgãos de fomento e outros. Em alguns casos podem haver URLs para sites nos artigos, principalmente nas notas de rodapé, mas o problema é que em grande parte dos casos é apenas texto e não link.

d) Popularidade: número de visitas. Nas revistas eletrônicas desenvolvidas com OJS isso pode ser dividido em visitas, que leem apenas o resumo, ou os que leem o artigo inteiro, pois muitas revistas implementam aplicativos que abrem o PDF no próprio site da revista.

Esses critérios possibilitam avaliar certas características das revistas, associando questões de produtividade (quantidade de artigos publicados), facilidade de acesso (por indexação por motores de busca), relacionamento com outros sites (inlinks e outlinks) e acessos. Assim, tornam-se indicadores úteis para verificar as revistas num ambiente complexo e pouco estruturado como a Web.

Da mesma forma, para ranquear repositórios utilizando indicadores webométricos, Aguillo et al. (2010) adaptaram algumas variáveis, de forma a ajustar a esses sistemas de informação. Avaliando esses indicadores sob o viés das revistas, na medida em que periódicos científicos e repositórios institucionais possuem unidades de informações baseadas em documentos digitais, pode-se ajustar como:

a) Tamanho (Size): Número de páginas indexadas em motores de busca, que facilita a recuperação. No caso das revistas implementadas com o OJS representam as páginas de informação das revistas, seus fascículos e artigos, visto que cada uma dessas páginas possuem uma URL distinta, inclusive os textos completos em PDF, XML, E-Pub e outros formatos.

b) Acadêmico (Scholar): número de páginas indexadas no google acadêmico. No caso das revistas desenvolvidas com o OJS, destaca-se a importância de configurar a revista para facilitar essa indexação, sendo que esse indicador restringe quase ao número de artigos publicados na revista.

c) Arquivos (PDF files): Esse indicador pode ser trocado pela quantidade de arquivos em vários formatos, visto que revistas podem disponibilizar os seus artigos em vários formatos, de forma a facilitar a leitura. Assim, revistas que disponibilizam os artigos em PDF, HTML, XML, E-Pub e outros podem ser melhor avaliadas.

d) Visibilidade (visibility): Número de inlinks, que pode ser ajustado a revista pelo WIF da revista, contemplando tanto as informações de citações, quanto de relacionamento com as redes e mídias sociais, blogs acadêmicos e portais de notícias.

Esses indicadores são úteis na avaliação de revistas como sistema de informação web, mas podem não atender a todas as especificidades dos periódicos, tais como a adesão ao DOI para identificação dos artigos ou do Orcid para identificação dos autores. Note que esses dois identificadores são links que são facilmente tratados por ferramentas webométricas.

O uso do DOI ainda não apresenta a totalidade, principalmente pelos custos, visto que grande parte das revistas são mantidas pelas universidades, institutos de pesquisa e associações, que possuem restrições orçamentárias. Com isso, restringe estudos webométricos baseados nesses identificadores, que são interlinks.

O Orcid, por sua vez, é gratuito para os autores e implementado como campo de descrição dos autores no OJS. Entretanto, ainda não apresenta grande adesão pelos pesquisadores brasileiros. O Orcid seria outro interlink importante para estudos webométricos relacionados a revista, pois relacionaria publicação, periódico e autor, com possibilidades de cruzamentos, verificação de colaboração e outros.

Cabe destacar que as revistas científicas desenvolvidas com o OJS possuem características 
múltiplas que permitem vários tipos de avaliações, ultrapassando a questão relacionada a citações. Por ser um sistema de informação web, um site, uma fonte de informação, entre outros, pode ser avaliado de várias formas, que em cada caso utiliza indicadores diferentes.

\section{CONSIDERAÇÕES FINAIS}

Métricas da web requerem precisão, abrangência e flexibilidade devido ao ambiente dinâmico e altamente mutável, com novas tecnologia sendo inseridas e algumas descontinuadas. Mesmo com pouco tempo, a web apresenta a complexidade e variedade de informação, de forma a estar se tornando a principal fonte de informação atual. Assim, a webometria, como sendo a métrica da web, pode se tornar um grande aliado no entendimento desse ambiente.

Assim, o presente estudo focou no uso dessa métrica nos periódicos científicos, mostrando-se eficaz para avaliação das relações da revista com o ambiente, mas não se apresentando totalmente adequado a todas as necessidades de avaliação. Nesse sentido, o estudo revela certo alinhamento com as conclusões de Santos e Kobashi (2009), no qual revela a necessidade de tornar os estudos métricos mais qualitativos, ou seja, com análise mais profunda dos indicadores.

Além do Fator de Impacto Web (WIF), a webometria pode ser útil em verificar indicadores mais atuais com o índice- $\mathrm{H}$ das revistas, na medida em que consegue levantar a produtividade e o uso dos artigos publicados. A utilização de técnicas e ferramentas webométricas são desenvolvidas para serem gerais e requerem adaptação para cada sistema de informação, visto as especificidades de cada um.

Nota-se que os estudos webométricos ainda requerem maiores pesquisas, de forma a se adaptar a todos os tipos de sistemas de informação, como as revistas, pois o uso de indicadores webométricos podem ser afetados por outros pontos como a de múltiplas cópias, visto que é comum um autor publicar um documento e depois depositá-lo em repositório ou em banco de documentos como o Researchgate. Com isso, cria um complicador na análise pura de links, requerendo que a webometria se torne mais abrangente, com estudos gerais na web.

Outro ponto a discutir é a maior difusão do Orcid como identificador do autor, visto que é um identificador e um link. Da mesma forma a adesão ao DOI, a utilização de padrões internacionais de identificação dos elementos constituintes das publicações facilitam a forma com que os links possam ser coletados, armazenados e analisados com maior acurácia, utilizando as métricas e ferramentas webométricas.

Nota-se ainda que estudos webométricos requer aprofundamento, na medida em que suas ferramentas precisam de evolução, dado a quantidade de informações existente nesse ambiente. Maina (2012) reflete que a webometria para periódicos científicos requerem estudos mais profundos, de aspectos qualitativo, de forma a obter melhores resultados. Com isso, desenvolver ou adaptar indicadores de forma a atender as especificidade dos periódicos científicos.

\section{WEBOMETRICS AND ELECTRONIC SCIENTIFIC JOURNAL}

ABSTRACT Metrics for evaluating scientific journals have been used by many disciplines, not only in librarianship or in information science, since journals are a communication channel used by all disciplines to disseminate research results. However, there is no consensus as to the best metric for its evaluation, given the disciplinary differences, technological evolution and changes in the publication processes. Thus, the present study aims to review the concepts and applications of webometry, within the framework of scientific journals. With this, contribute to the current discussion on indicators. The study reveals that the inlinks of an online electronic journal are largely quotations, aligning bibliometrics, but may also be an indication of articles in social networks, approaching altimetry. However, studies based only on the links do not provide the necessary accuracy to the metrics, so that deeper qualitative studies are required for the development of better indicators, given the problems involving the links.

Keywords: $\quad$ Bibliometrics. Web magazine. Metric studies. Scientific magazines. webometric indicators. 


\section{REFERÊNCIAS}

AGUILLO, I. F.; ORTEGA, J. L.; FERNÁNDEZ, M. Webometric ranking of world universities: Introduction, methodology, and future developments. Higher education in Europe, v. 33, n. 2-3, p. 233244, 2008. Disponível em: <http://www.tandfonline.com/doi/abs/10.1080/03797720802254031> Acesso em 13 abr. 2017.

AGUILLO, I. F. et al. Indicators for a webometric ranking of open access repositories. Scientometrics, v. 82, n. 3, p. 477-486, 2010. Disponível em: <https://link.springer.com/ article/10.1007/s11192-010-0183-y > Acesso em 13 abr. 2017.

AGUILLO, I. F. Is Google Scholar useful for bibliometrics? A webometric analysis. Scientometrics, v. 91, n. 2, p. 343-351, 2011. Disponível em: <https://link.springer.com/ article/10.1007/s11192-011-0582-8> Acesso em 14 abr. 2017

ALMIND, T. C.; INGWERSEN, P. Informetric analyses on the world wide web: methodological approaches to 'webometrics'. Journal of documentation, v. 53, n. 4, p. 404-426, 1997. Disponível em: <http://www.emeraldinsight. com/doi/abs/10.1108/EUM0000000007205> Acesso em 14 mar. 2017

BJÖRNEBORN, L.; INGWERSEN, P. Perspectives of webometrics. Cientometrics, v. 50, n. 1, p. 6582, 2001.Disponível em: <https://link.springer. com/article/10.1023/A:1005642218907> Acesso em 20 mar. 2017

COSTA, T, et al. A Bibliometria e a Avaliação da Produção Científica: indicadores e ferramenta. In: CONGRESSO NACIONAL DE BIBLIOTECÁRIOS, ARQUIVISTAS E DOCUMENTALISTAS, 11., 2012, Lisboa. Anais... . Lisboa: BAD, 2012. Disponível em: <http://www.bad.pt/publicacoes/index.php/ congressosbad/article/view/429/pdf $>$. Acesso em: 24 abr. 2017.

CRUZ, A. A. A. C. da et al. Impacto dos periódicos eletrônicos em bibliotecas universitárias. Ci. Inf, v. 32, n. 2, p. 47-53, 2003. Disponível em: <http:// revista.ibict.br/ciinf/article/view/1004>

Acesso em: 24 maio 2017.

IBRAHIM, G. AF. Theory and Practice of the Webometrics Discipline. In: ANNUAL CONFERENCE - HUMANITIES AND EDUCATIONAL STUDIES, 2013, Cartum. Proceedings... . Cartum: Uofk, 2013.Disponível em: $\quad<$ http://khartoumspace.uofk.edu/ handle/123456789/9709> . Acesso em: 24 abr. 2017.

INGWERSEN, P. Reflections on Receiving the ASIS\&T 2016 Award of Merit. Bulletin of the Association for Information Science and Technology, v. 43, n. 2, p. 13-14, Dec./Jan 2017. Disponível em: <http://onlinelibrary.wiley. com/doi/10.1002/bul2.2017.1720430207/full> Acesso em 30 mar. 2017

The calculation of web impact factors. Journal of documentation, v. 54, n. 2, p. 236243, 1998. Disponível em: <http://www. emeraldinsight.com/doi/abs/10.1108/ EUM0000000007167>. Acesso em 30 abr. 2017

KOUSHA, K.; THELWALL, M. News stories as evidence for research? BBC citations from articles, books and Wikipedia. Journal of the Association for Information Science and Technology, 2017a. Disponível em: <https://www.researchgate. net/publication/313250748_News_stories_as evidence_for_research_BBC_citations_from articles_books_and_Wikipedia>. Acesso em 30 abr. 2017

Are Wikipedia citations important evidence of the impact of scholarly articles and books?. Journal of the Association for Information Science and Technology, v. 68, n. 3, p. 762-779, Marc. 2017b. Disponível em: <http:// onlinelibrary.wiley.com/doi/10.1002/asi.23694/ full>. Acesso em 30 abr. 2017

LORENTZEN, D. G. Webometrics benefitting from web mining? An investigation of methods and applications of two research fields. Scientometrics, v. 99, n. 2, p. 409-445, 2014. Disponível em: <https://link.springer.com/ article/10.1007/s11192-013-1227-x>. Acesso em 30 abr. 2017 
LOOSE; E. B.; Lima, M. R. D. V. A ciência nos portais de notícias: notas para pensar a popularização científica a partir do jornalismo online. ANIMUS - Revista Interamericana de Comunicação Midiática, v 12, n. 23, 2013. Disponível em: <http://dx.doi. org/10.5902/217549779085>. Acesso em 21 abr. 2017

MAINA, S. Webometrics and journal websites. European Science Editing, v. 38, n. 3, 2012. Disponível em: <http://europeanscienceediting. eu/articles/webometrics-and-journalwebsites/>. Acesso em 21 abr. 2017

MANCA, S.; RANIERI, M. Networked Scholarship and Motivations for Social Media use in Scholarly Communication. The International Review of Research in Open and Distributed Learning, v. 18, n. 2, 2017. Disponível em: <http://www.irrodl.org/index.php/irrodl/ article/view/2859>. Acesso em 21 abr. 2017

MEADOWS, A. J. Os periódicos científicos e a transição do meio impresso para o eletrônico. Revista de Biblioteconomia de Brasília, v. 25, n. 1, p. 5-14, 2001. Disponível em: <http://www. brapci.ufpr.br/brapci/_repositorio/2010/10/ pdf_29f176742d_0012269.pdf>. Acesso em 26 mar.. 2017

NASCIMENTO, A. G.; ODDONE, N. Uso de altmetrics para avaliação de periódicos científicos brasileiros em ciência da informação. Ciência da Informação em Revista, v. 2, n. 1, p. 3-12, 2015. Disponível em: <http://www.seer.ufal.br/index. $\mathrm{php} / \mathrm{cir} /$ article/view/1745>. Acesso em 18 mar.. 2017

NG; K. Student-run Academic Journals: A New Trend in Scholarly Communication. In: 2017 AAAS Annual Meeting (February 16-20, 2017). AAAS, 2017. Disponível em: <https:// aaas.confex.com/aaas/2017/webprogram/ Paper19950.html>. Acesso em 18 mar.. 2017

NINKOV, A; VAUGHAN, L. A webometric analysis of the online vaccination debate. Journal of the Association for Information Science and Technology, 2017. Disponível em: <http:// onlinelibrary.wiley.com/doi/10.1002/asi.23758/ full>. Acesso em 18 mar.. 2017

NORUZI, A. The web impact factor: a critical review. The electronic library, $v$. 24, n. 4, p. 490-500, 2006. Disponível em: <http://www.emeraldinsight.com/doi/ abs/10.1108/02640470610689188>. Acesso em 25 mar.. 2017

PORTO, C. de M. Impacto da internet na difusão da cultura científica brasileira: as transformações nos veículos e processos de disseminação e divulgação científica. Tese de Doutorado. 2013. Disponível em: <http://repositorio.ufba.br/ri/ handle/ri/9038>. Acesso em 12 mai. 2017.

SANTOS, R. N. M. dos; KOBASHI, N. Y. Bibliometria, cientometria, infometria: conceitos e aplicações. Pesq. bras. Ci. Inf., Brasília, v.2, n.1, p.155-172, jan./dez. 2009. Disponível em: <http://inseer.ibict.br/ancib/index.php/tpbci/ article/view/21/43> Acesso em 10 abr. 2017.

SHEMA, H.; BAR-ILAN, J.; THELWALL, M. Scholarly blogs are a promising altmetric source. Value of bibliometrics, v. 37, jun. 2014. Disponível em: <https://www.researchtrends. com/issue-37-june-2014/scholarly-blogs-are-apromising-altmetric-source/> Acesso em 15 mai. 2017.

SHEMA, H.; BAR-ILAN, J.; THELWALL, M. Do blog citations correlate with a higher number of future citations? Research blogs as a potential source for alternative metrics. Journal of the Association for Information Science and Technology, v. 65, n.5, p. 1018-1027, 2014. Disponível em: <http://onlinelibrary.wiley. com/doi/10.1002/asi.23037/abstract> Acesso em 12 abr. 2017

SHINTAKU, M.; BRITO, R. F.; CARVALHO NETO, S. A avaliação dos portais de revistas brasileiros implementados com o SEER/OJS por meio do levantamento da indexação pelo Latindex e SciELO. Informação \& Sociedade: Estudos, v. 24, n. 2, 2014. Disponível em: <http://www.ies.ufpb.br/ojs/index.php/ies/ article/view/18671> Acesso em 10 abr. 2017. 
SILVA, J. A. da; BIANCHI, M. de L. P. Cientometria: a métrica da ciência. Paidéia (Ribeirão Preto), v. 11, n. 21, p. 5-10, 2001. Disponível em: <http://www.scielo.br/ scielo.php?script $=$ sci_arttext\&pid $=$ S0103863X2001000200002>. Acesso em 28 abr. 2017.

STUART, E.; STUART, D.; THELWALL, M. An investigation of the online presence of UK universities on Instagram. Online Information Review, 2017. Disponível em: $<\underline{w l v}$. openrepository.com/wlv/handle/2436/620460> Acesso em 28 abr. 2017

STUMPF, I. C. Passado e futuro das revistas científicas. Ciência da Informação, v. 25, n. 3, 1996. Disponível em: <http://revista.ibict.br/ ciinf/article/view/637> Acesso em 28 abr. 2017

THELWALL, M. Bibliometrics to webometrics. Journal of information science, v. 34, n. 4, p. 605-621, 2008. Disponível em: <http://journals.sagepub.com/doi/ abs $/ 10.1177 / 0165551507087238>$. Acesso em 27 abr. 2017

\begin{abstract}
THELWALL, M.; VAUGHAN, L.; BJÖRNEBORN, L. Webometrics. ARIST, v. 39, n. 1, p. 81-135, 2005. Disponível em: <http://onlinelibrary.wiley.com/doi/10.1002/ aris.1440390110/abstract>. Acesso em 27 abr. 2017
\end{abstract}

VANTI, N. A. P. Da bibliometria à webometria: uma exploração conceitual dos mecanismos utilizados para medir o registro da informação e a difusão do conhecimento. Ciência da informação, v. 31, n. 2, p. 152-162, 2002. Disponível em: <http://www.scielo.br/scielo. php?pid=S0100-19652002000200016\&script $=$ sci abstract\&tlng $=\mathrm{pt}>$. Acesso em 27 maio 2017

ZAHRAN, D. I. et al. A comparative approach to web evaluation and website evaluation methods. International Journal of Public Information Systems, v. 10, n. 1, 2014. Disponível em: <http:// www.ijpis.net/ojs/index.php/IJPIS/ article/view/126>. Acesso em 16 maio 2017 